\title{
Lung manifestation and pulmonary hypertension in systemic lupus erythematodes
}

\author{
Kaveh Akbari $^{1 *}$, Franz A. Fellner ${ }^{1,2}$, David Lang ${ }^{3}$ and Julia Röper-Kelmayr ${ }^{1}$ \\ ${ }^{1}$ Central Radiology Institute, Kepler University Hospital, Medical Faculty of the Johannes Kepler University, Linz, Austria \\ ${ }^{2}$ Medical Faculty of the Friedrich-Alexander-University of Erlangen-Nürnberg, Germany \\ ${ }^{3}$ Department for Pulmonary Medicine, Kepler University Hospital, Medical Faculty of the Johannes Kepler University, Linz, Austria
}

A 34 year old male patient was referred for assessment of chronic cough since five years, exertional dyspnea and weight loss of $10 \mathrm{~kg}$ in two years. The patient was a non-smoker without allergenic exposition.

Spirometry showed a primary restriction with disproportionate restriction of the vital capacity and severe reduced gas transfer.

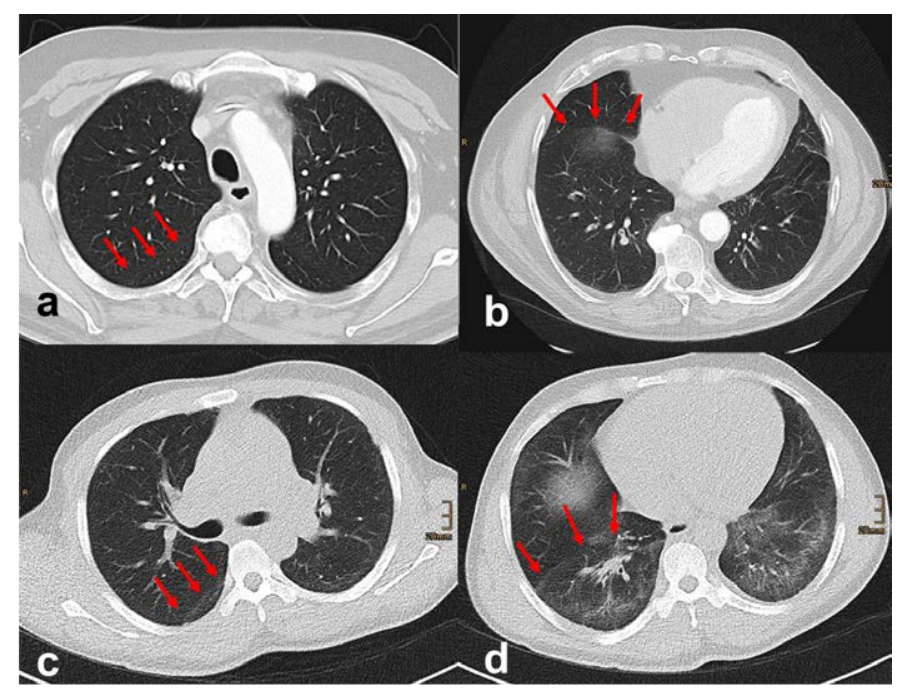

Figure 1. $\mathrm{a}+\mathrm{b}$ : normal volume of the lower lobes. $\mathrm{c}+\mathrm{d}$ : patient with volume loss in both lower lobes. Volume loss in lower lobes is inferred from the position of the oblique fissures on axial high-resolution CT imaging. The oblique fissure normally originates posteriorly at the level of the aortic arch (a), and nearly touches the anterior chest wall when the diaphragm is reached (b). In this case the oblique fissure originates posteriorly at the level of the carina (c) and is set clearly back at the level of the diaphragm (d) due to volume loss of the lower lobes.

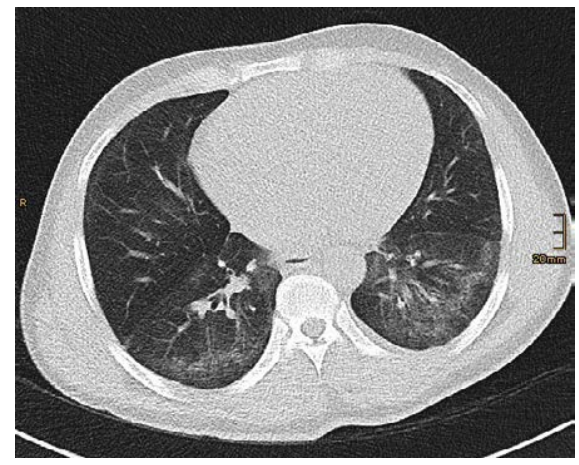

Figure 2. Fine reticulation in a basal distribution with immediate subpleural sparing is relatively specific sign for the NSIP pattern in high-resolution CT.
A high-resolution CT of the lung was performed, which revealed volume loss (Figure 1), fine reticulation in a basal distribution with subpleural sparing, characterized by a narrow zone of peripheral relatively normal lung (Figure 2) and mild traction bronchiectasis (Figure 3) consistent with fibrosis with non-specific interstitial pneumonia (NSIP) pattern.

Furthermore, the pulmonary trunk showed a maximal diameter of $40 \mathrm{~mm}$ being suspicious of mild pulmonary hypertension which was proved in the pulmonary artery catheterization.

Considering the above mentioned findings, further assessment regarding a rheumatic spectrum disorder was performed and systemic lupus erythematodes was diagnosed.

The high-resolution CT of the lungs is the only appropriate imaging modality for differentiating lung fibrosis [1]. The lung involvement of rheumatic disease presents typically with the NSIP pattern, its manifestation in high-resolution CT includes volume loss with a predominance in the lower lobes, ground-glass opacities, reticular opacities and irregular linear opacities with immediate subpleural sparing as a relatively specific sign and traction bronchiectasis associated with NSIP[2].

Pulmonary hypertension can also be related with rheumatic

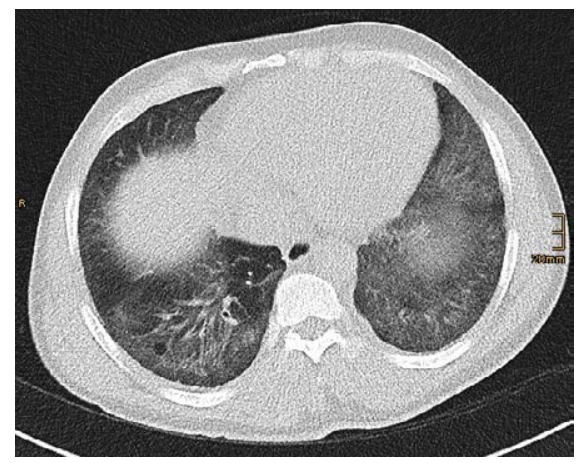

Figure 3. Mild traction bronchiectasis associated with fibrotic NSIP.

Correspondence to: Kaveh Akbari, Central Radiology Institute, Kepler University Hospital, Medical Faculty of the Johannes Kepler University, Linz, Austria, E-mail: kaveh.akbari@kepleruniklinikum.at

Received: July 10, 2017; Accepted: July 24, 2017; Published: July 26, 2017 
Akbari K (2017) Lung manifestation and pulmonary hypertension in systemic lupus erythematodes

diseases[3]. When it is presented together with lung fibrosis with NSIP pattern a rheumatic spectrum disorder should be considered.

\section{References}

1. Jacob J, Hansell DM (2015) HRCT of fibrosing lung disease. Respirology 20: 859-872. [Crossref]
2. Kligerman SJ, Groshong S, Brown KK, Lynch DA (2009) Nonspecific interstitial pneumonia: radiologic, clinical, and pathologic considerations. Radiographics 29: 73-87.

3. Distler O, Pignone A (2006) Pulmonary arterial hypertension and rheumatic diseases from diagnosis to treatment. Rheumatology (Oxford) 45: iv22-25.[Crossref]

Copyright: (O2017 Akbari K. This is an open-access article distributed under the terms of the Creative Commons Attribution License, which permits unrestricted use, distribution, and reproduction in any medium, provided the original author and source are credited. 